\title{
Large scale Single-crystal Cu(In,Ga)Se 2 Nanotip Arrays For High Efficiency Solar Cell
}

Chin-Hung Liu ${ }^{1, \dagger}$, Chia-Hsiang Chen ${ }^{1, \dagger}$, Szu-Ying Chen ${ }^{1}$, Yu-Ting Yen ${ }^{1}$, Wei-Chen Kuo ${ }^{2}, Y u-K u a n g ~ L i a o^{3}$, Jenh-Yih Juang ${ }^{2}$, Hao-Chung Kuo ${ }^{3}$, Chih-Huang Lai ${ }^{1}$, Lih-Juann Chen ${ }^{1}$,Yu-Lun Chueh ${ }^{1, *}$

${ }^{1}$ Department of Materials Science and Engineering, National Tsing Hua University, HsinChu, 30013, Taiwan, R.O.C.

${ }^{2}$ Department of Electrophysics, National Chiao Tung University, HsinChu, 30013, Taiwan, R.O.C.

3.Department of photonics, National Chiao Tung University, HsinChu, 30013, Taiwan, R.O.C.

${ }^{\dagger}$ These authors contributed equally to this work.

* Correspondence should be addressed to Y.L.C. (ylchueh@mx.nthu.edu.tw)

\section{Supplementary Information}




\section{Methods Summary}

Sputtering process of CIGS thin film without post-selenization process. The CIGS layers were fabricated by using a sputtering system with four 2-inch magnetron sputtering guns and a rotatable substrate holder with heating apparatus. Back molybdenum electrode with thickness of $1 \mu \mathrm{m}$ was deposited onto pre-cleaned $2 \times 2 \mathrm{~cm}^{2}$ soda-lime glass (SLG) substrate by DC magnetron sputtering. CIGS films with the chalcopyrite phase were deposited by direct sputtering of a single quaternary CIGS target. The CIGS quaternary target has a composition of $\mathrm{Cu}, \mathrm{In}, \mathrm{Ga}$, and $\mathrm{Se}$ of $25,17.5,7.5$, and 50 at $\%$, respectively. The sputtering chamber was pumped down to a base pressure of $1 \times 10^{-6}$ Torr before deposition. The working pressure during the deposition was 3 mTorr with pure Ar gas. CIGS films were deposited by pulse DC magnetron sputtering at $500{ }^{\circ} \mathrm{C}$.

Formation of CIGS NTRs by ion milling process. Ion milling system with 4-inch working area was applied to create CIGS nanotip arrays. The incident angle of ion beams can be adjusted from $90^{\circ}$ to $15^{\circ}$ manually. Pre-milling base pressure was pumped down to $3 \times 10^{-6}$ Torr. Working pressure during milling was fixed at $1.2 \times 10^{-4}$ Torr. Filament current was set at $3.85 \mathrm{~A}$ with a cathode current of $0.76 \mathrm{~A}$ while beam current and accelerator current were set at $50 \mathrm{~mA}$ and $0.76 \mathrm{~mA}$, respectively. Beam voltage and accelerator voltage were set at $400 \mathrm{~V}$ and $300 \mathrm{~V}$, respectively.

Fabrication of CIGS solar cell. After creating nanostructure on the surface of CIGS, $100 \mathrm{~nm}$-thick CdS buffer layer was prepared by chemical bath deposition (CBD) with the mixed solution composition of Cd $=1.5 \mathrm{mM}$, Thiourea $\left(\mathrm{CS}\left(\mathrm{NH}_{2}\right)_{2}\right)=75 \mathrm{mM}$, and $\mathrm{NH}_{4} \mathrm{OH}=0.924 \mathrm{mM}$, respectively. A $200 \mathrm{~nm}$-thick transparent conductive $\mathrm{Al}: \mathrm{ZnO}$ layer (TCO) was deposited by RF magnetron sputtering. E-gun evaporation with contact mask was applied to deposit the Al top contact with cell size of $0.5 \mathrm{~cm}^{2}$.

Characterization. Crystal structures of the CIGS films were characterized by a Shimadzu X-ray diffractometer (XRD) and Grazing Incident Angle XRD (GIAXRD) with $\mathrm{Cu} \mathrm{K}_{\alpha}(\lambda=0.154 \mathrm{~nm})$ as the 
radiation source. Morphologies and microstructures were studied by field-emission scanning electron microscopy (FE-SEM, JSM-6500F, JEOL) and transmission electron microscopy (TEM, JEM-3000F, JEOL). The density of CIGS NTRs was examined by atomic force microscopy (AFM, Di-3000, Vecco). Composition of CIGS NTRs was characterized with an energy dispersive spectrometer (EDS) attached to the TEM. Optical behaviors of CIGS NTRs were examined by UV-VIS-NIR spectrometer (UV-4100, Hitachi). The current-voltage characteristics and external quantum efficiency of photovoltaic devices were measured by Keitheley 4200 analyzer under AM 1.5 solar illumination with the power density of $100 \mathrm{~mW} / \mathrm{cm}^{2}$ at $25^{\circ} \mathrm{C}$ using helium light as the light source. 


\section{Evolution of CIGS NTRs length at different milling angles and time}

Figure S1 shows the average lengths of CIGS NTRs under different milling time. The average length of CIGS NTRs are $\sim 140, \sim 160, \sim 320, \sim 190$, and $\sim 120 \mathrm{~nm}$ at milling time of 10, 20, 30, 60, and $90 \mathrm{~min}$. respectively. Grazing incident angle x-ray diffraction pattern (GIAXRD) results illustrated in Figs. S1b and S1c indicate appearance of copper or copper-rich CIGS phases once milling time was longer than 10 min. Characteristic peaks of CIGS located at $27.04^{\circ}, 44.84^{\circ}$, and $53.16^{\circ}$, corresponding to (220)/(204), (112), and (116)/(312) planes can be indexed, respectively. In addition, characteristic peak of $\mathrm{Cu}(111)$ located at $43.37^{\circ}$ was found.
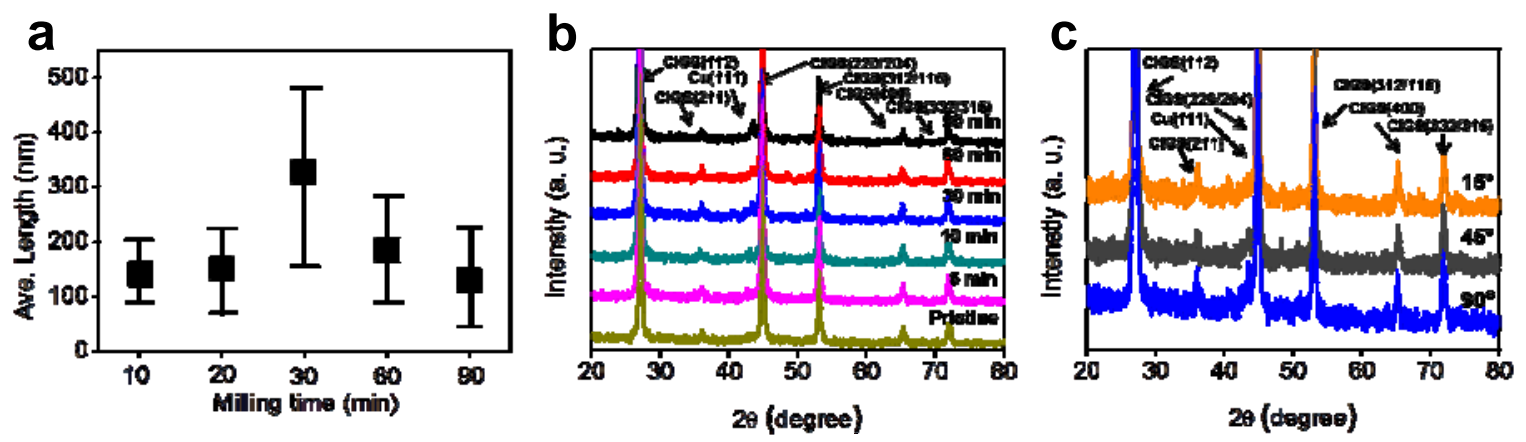

Figure S1: a, Statistic results of length distribution at different milling time from 10 to $90 \mathrm{~min}$, respectively. b, Grazing incident angle XRD results of CIGS NTRs with milling angle of $90^{\circ}$ at different milling time. c, Grazing incident angle XRD results of CIGS NTRs with different angles at fixed milling time of $30 \mathrm{~min}$. 
$\underline{\text { AES mapping results of copper segregation, secondary phase, or copper-rich CIGS after } \mathrm{Ar}^{+}}$ milling process

We use the 2-D AES (Auger Electron Spectrum) mapping to confirm evidence of $\mathrm{Cu}$ segregation, secondary phase, or Cu-rich CIGS phase out of surface of CIGS NTRs after ion milling applied. As can be seen that no segregation of $\mathrm{Cu}, \mathrm{Ga}$, In, and Se were found before ion milling while distinct segregation of $\mathrm{Cu}, \mathrm{In}, \mathrm{Se}$, and $\mathrm{Ga}$ can be found after 2 min milling.

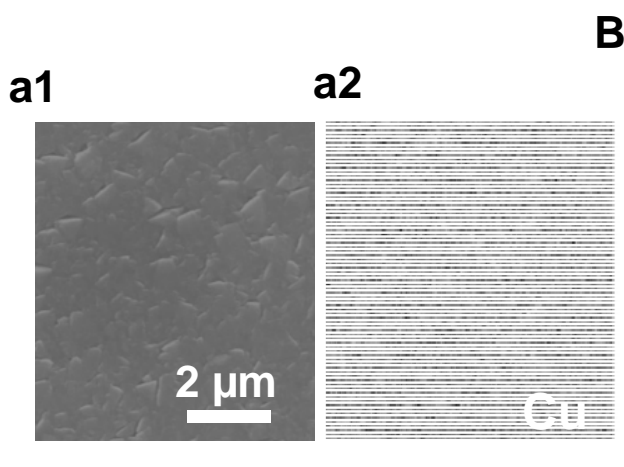

Before lon milling
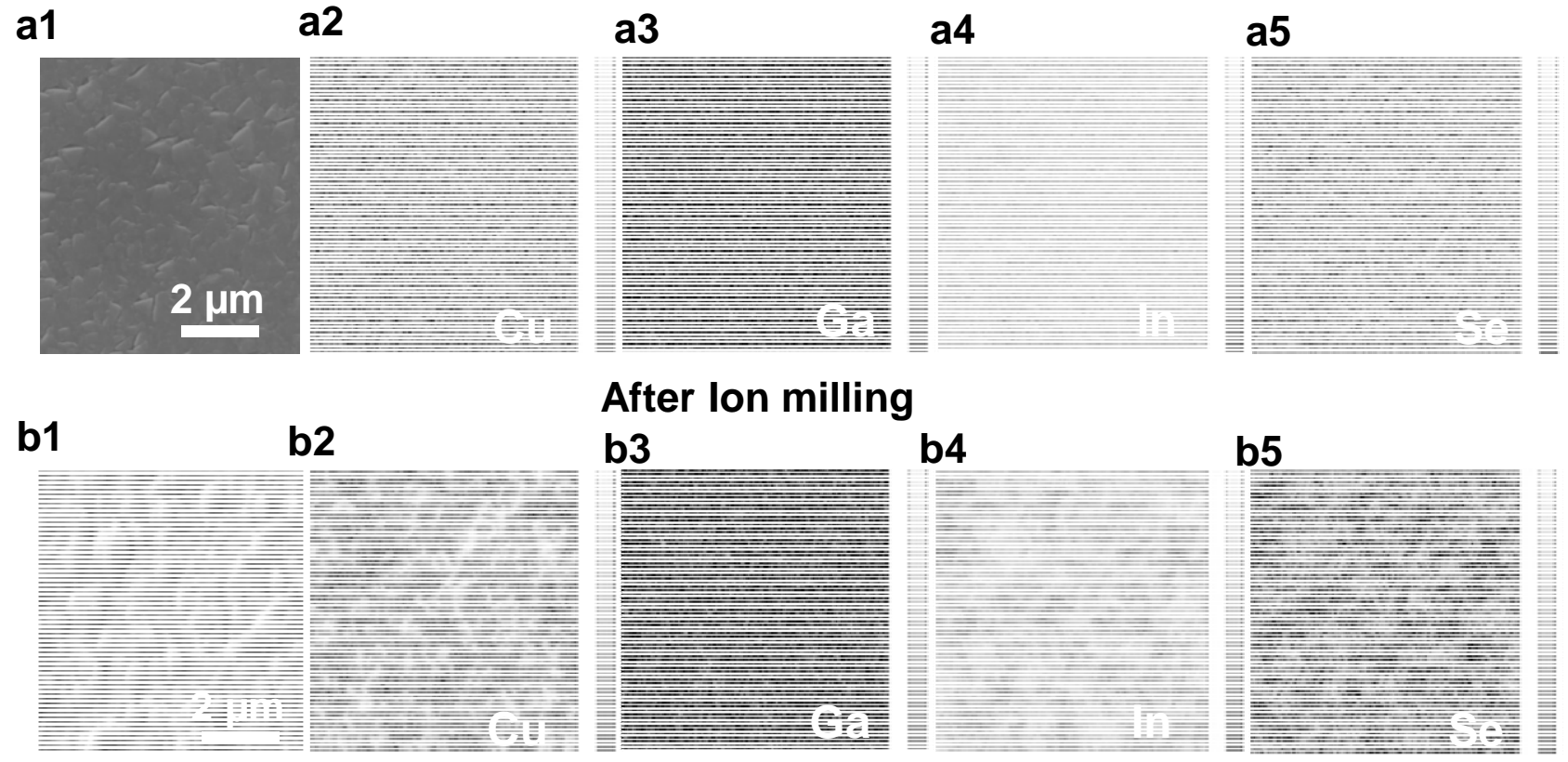

\section{After lon milling}

b3

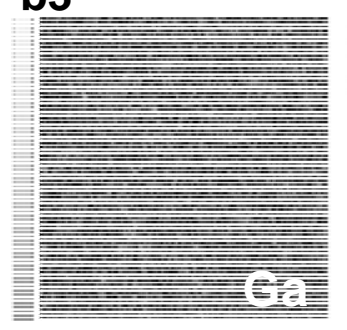

b4

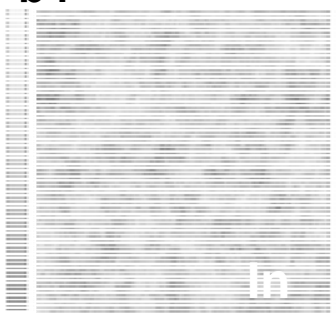

b5

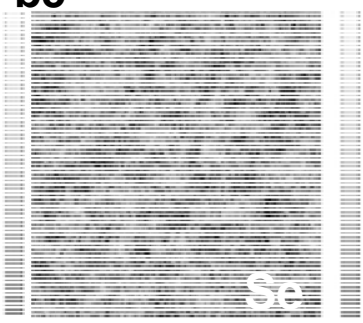

Figure S2 a1, a 5,: 2-D AES mapping of as-sputtered CIGS thin film without ion milling. b1, b5,: 2-D AES mapping of CIGS thin film after 2 min milling. 


\section{Criteria for the formation of CIGS NTRs}

The formation criteria for nanostructure by ion milling processes were reported to mainly depend on surface roughness and redistributions of sputtering yield with different elements. However, the crystal structure and crystalline quality were found to play important roles for creating nanostructures. The criteria for formation of CIGS NTRs by ion milling process were resulted from (i) different surface curvature due to surface roughness, (ii) multiple components, and (iii) good crystal quality, thereby enabling highly anisotropic milling effect. Figure S1 shows the XRD results for three kinds of CIGS crystal structures, namely, crystal CIGS thin film, amorphous CIGS thin film, and re-crystallized CIGS thin film after annealing treatment at $550^{\circ}$. Figures $3 \mathrm{Sb}$ and $3 \mathrm{Sc}$ shows $\mathrm{SEM}$ images of $\mathrm{AZO}$ and $\mathrm{Cu}_{2} \mathrm{Se}$ thin film after ion milling for $15 \mathrm{~min}$. Note that no nanostructure was created after ion milling.

a

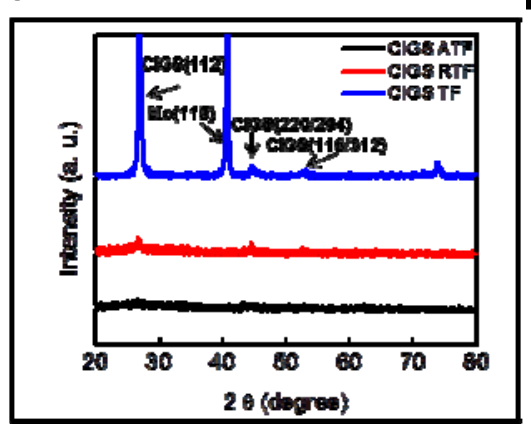

b

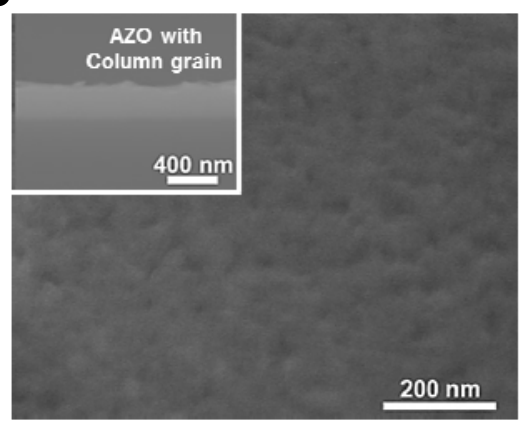

C

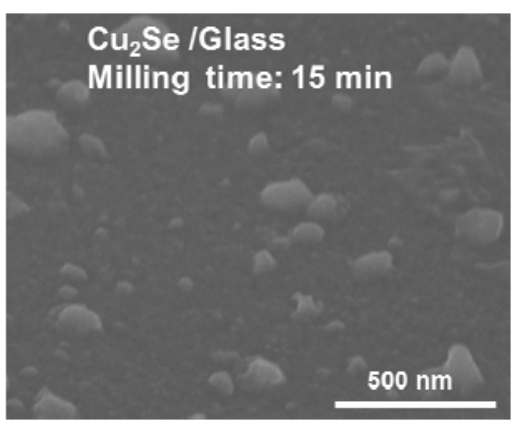

Figure S3: a, XRD results for three kinds of CIGS samples from Figs. 3. b, SEM image of AZO thin film after ion milling at $90^{\circ}$ for $15 \mathrm{~min}$. Inset show the cross-section SEM image, revealing that no CIGS nanostructure can be formed. c, the milling result of $\mathrm{Cu} 2 \mathrm{Se}$ at $90^{\circ}$ for $30 \mathrm{~min}$. 


\section{$\underline{\text { conditions }}$}
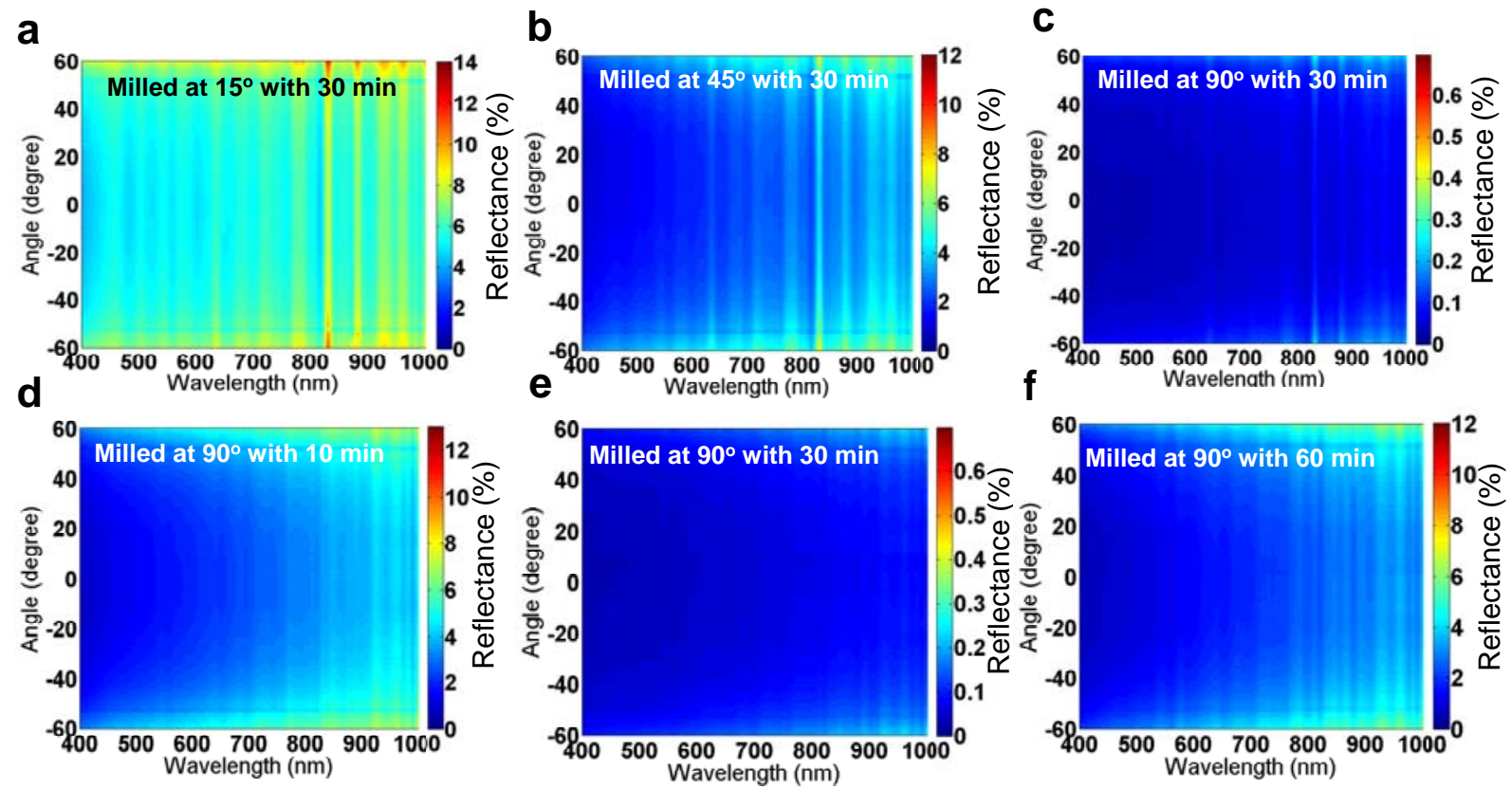

Figure S4: 2D reflectance mappings at incident angles from $-60 \mathrm{o}$ to $60 \mathrm{o}$ with incident light wavelengths from 400-1000 nm for the CIGS NTRs at milling angles of $\mathbf{a}, 90^{\circ}, \mathbf{b}, 45^{\circ}$, and $\mathbf{c}, 15^{\circ}$ for 30 minutes, respectively. d, to f, $2 \mathrm{D}$ reflectance mappings at ion milling angle of $90^{\circ}$ for 10,30 , and $60 \mathrm{~min}$, respectively. 


\section{Morphologies and crystal qualities of CIGS NTRs after KCN washing}

$\mathrm{KCN}$ washing was reported the best way to remove the secondary phase, such as $\mathrm{Cu}_{2} \mathrm{Se}$ cluster, $\mathrm{Cu}$-rich CIGS, and some defects on the surface of $\mathrm{CIGS}^{1,2}$. Figures S5a to S5e show the SEM images of CIGS NTRs without $\mathrm{KCN}$ washing and with $\mathrm{KCN}$ washing at different time. It can be seen that the shape and length of CIGS NTRs are changed after KCN washing, as shown in Figure S5f. Figure S5g shows the corresponding XRD results at different $\mathrm{KCN}$ washing time. Clearly, the excess $\mathrm{Cu}$ can be effectively removed after $\mathrm{KCN}$ washing over 7 min.

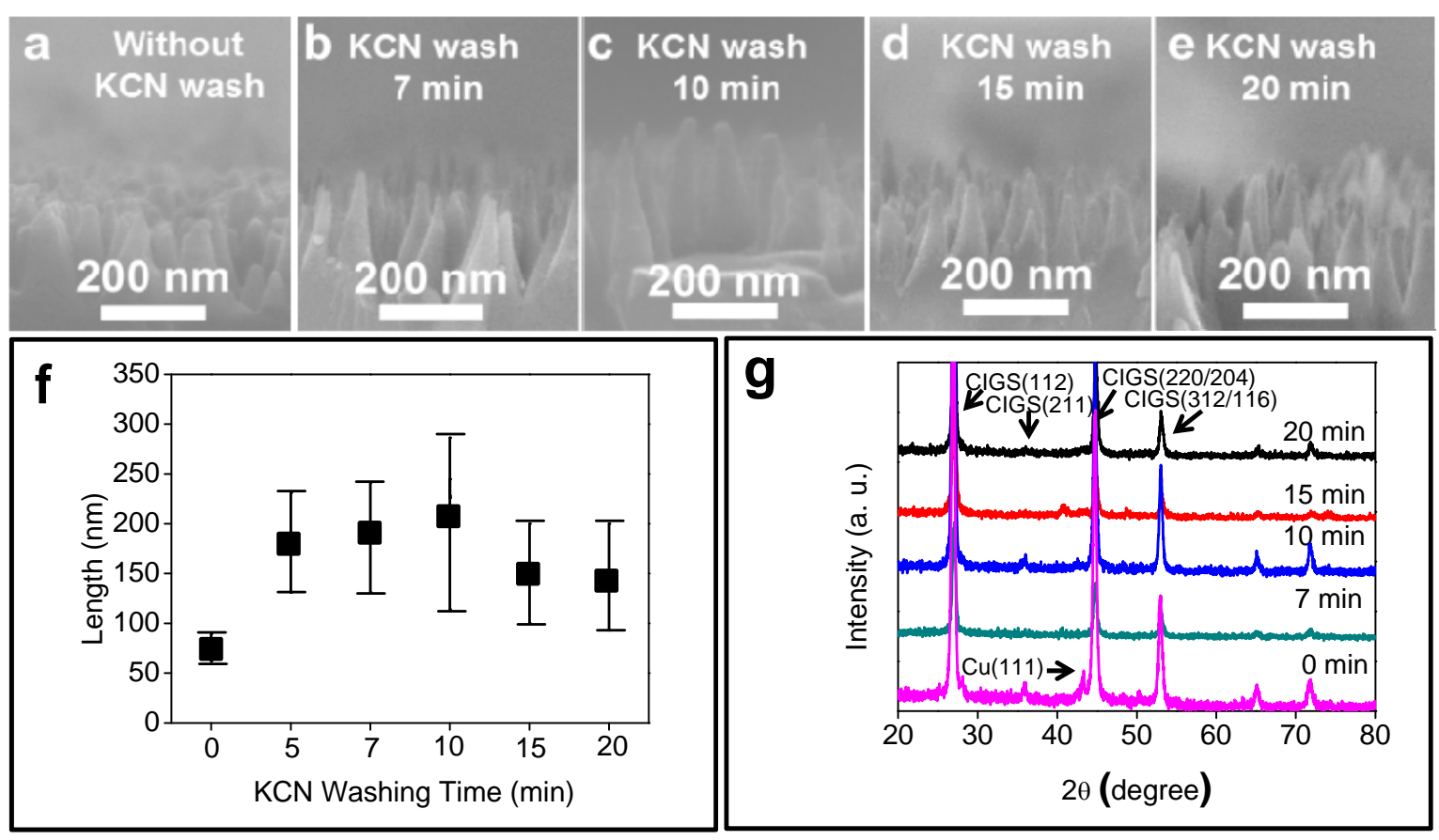

Figure S5: SEM images of CIGS NTRs without a, KCN washing and after KCN washing at b, 7 min, $\mathbf{c}$, $10 \mathrm{~min}$, d, $15 \mathrm{~min}$, and e, $20 \mathrm{~min}$, respectively. f, The statistic results in length of CIGS NTRs after KCN washing at different washing time. g, GIAXRD results of CIGS NTRs after KCN washing. 


\section{Coverage of chemical bath deposited (CBD) CdS buffer layer onto CIGS NTRs}

Well-developed chemical bath deposition is applied for coating of CdS buffer layer onto CIGS NTRs. In order to preserve the morphology of CIGS NTRs, CBD process had been optimized, with which the thickness of CdS layer was found to be $\sim 100 \mathrm{~nm}$. The crystal quality of CdS is confirmed with GIAXRD, which indicates nanocrystalline of the CdS layer.
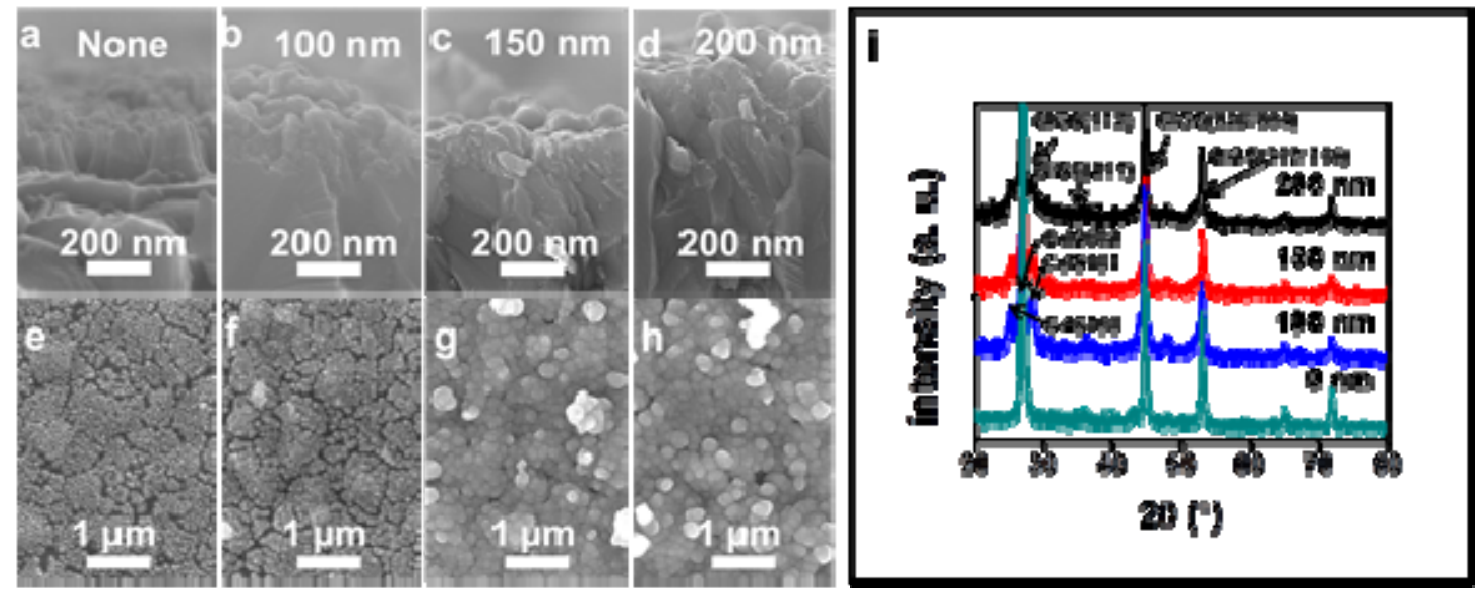

Figure S6: SEM images of different coverage with different thickness of CdS layers. a $\sim \mathrm{d}$, Cross section views of CdS/CIGS NTRs with CdS of 0, 100, 150, and $200 \mathrm{~nm}$, respectively. e $\sim$, Top views of CdS/CIGS NTRs with CdS of 0, 100, 150, and $200 \mathrm{~nm}$, respectively. g, GIAXRD spectra of CdS/CIGS NTRs. 


\section{n-type CdS buffer layer}
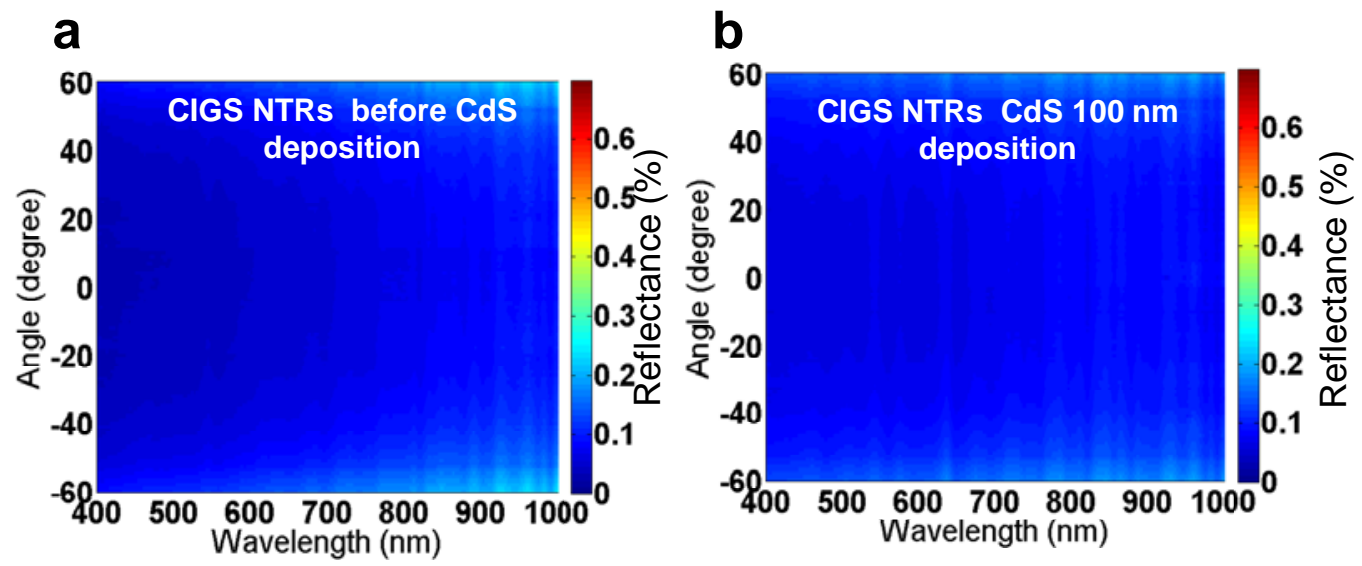

Figure S7: 2D reflectance mappings of CIGS NTRs a, before and $\mathbf{b}$, after coating of $100 \mathrm{~nm}$-thick CdS buffer layer. 


\section{Efficiency of CIGS NTRs solar cell at different KCN washing time}

In order to optimize the performance of CIGS NTRs solar cell, KCN wash time was set at $0,3,10,15$ and 20 min with the thickness of CdS buffer layer $\sim 100 \mathrm{~nm}$ as shown in Figure S8a. Inset shows I-V measurements of CIGS NTRs via two terminal electrodes. The extraordinary conductive behavior for asformed CIGS NTRs can be found, which is due to the formation of metallic secondary phase, excess $\mathrm{Cu}$ segregation, or $\mathrm{Cu}$-rich $\mathrm{CIGS}$ after ion milling. After $\mathrm{KCN}$ washing over 3 min, the high resistive behavior can be achieved, indicating that these metallic secondary phase, excess $\mathrm{Cu}$ segregation, or $\mathrm{Cu}-$ rich CIGS can be totally removed. In present case, after KCN washing of 20 min, we can achieve the CIGS NTRs solar cell with the highest efficiency of $\sim 5.2 \%$ (Figure S8b).

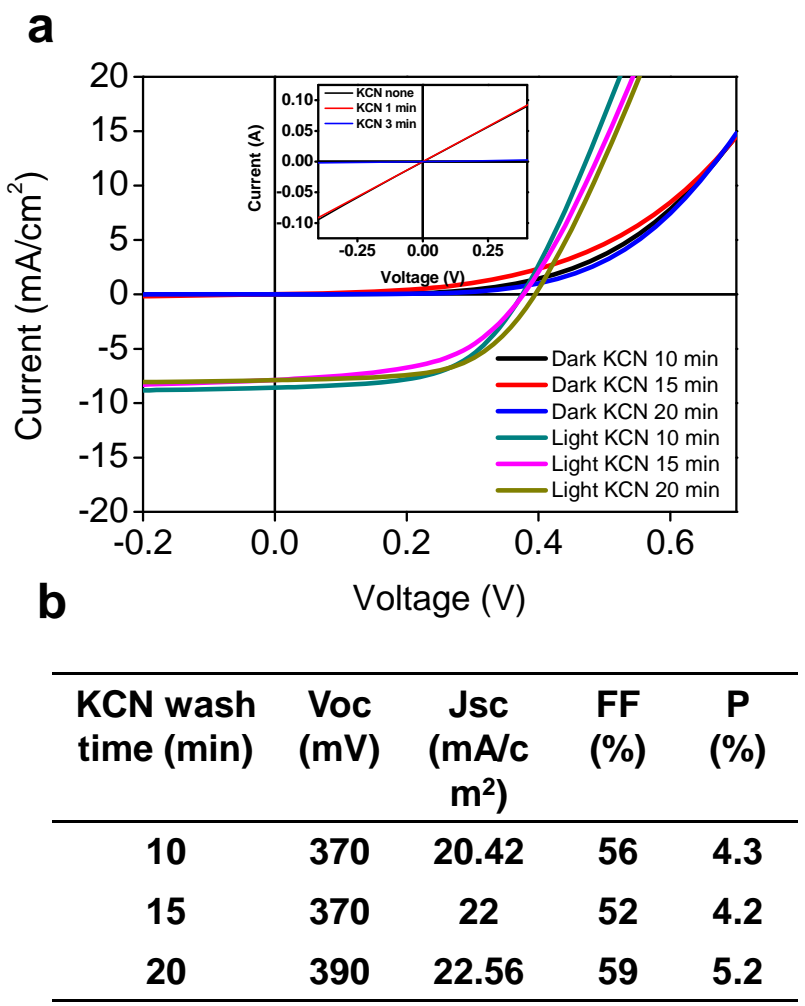

Figure S8: a, Dark and Light I-V measurements of CIGS NTRs solar cells at different KCN washing time from 10, 15 and 20 minutes, respectively. Inset shows the typical I-V behaviors of CIGS NTRs without $\mathrm{KCN}$ washing and with $\mathrm{KCN}$ washing for 1 and 3 min, respectively. b, The corresponding $\mathrm{V}_{\mathrm{oc}}, \mathrm{J}_{\mathrm{sc}}$, Efficiency, and filling factor of CIGS NTRs solar cell after KCN wash time for 10, 15 and 20 minutes, respectively. 


\section{Efficiency of CIGS NTRs solar cell at different thickness of n-type CdS buffer layer}

The performance of CIGS solar cell devices can be greatly enhanced by improving the interface between CIGS NTRs and n-type CdS buffer layer. To shed light on this point, different thickness of CdS layers from 50 to $150 \mathrm{~nm}$ were used in order to fully cover the CIGS NTRs as shown in Figure S8 where the 20 min $\mathrm{KCN}$ washing was used to removed excess $\mathrm{Cu}$ segregation, metallic secondary phase, and $\mathrm{Cu}-$ rich CIGS. The thickness of $50 \mathrm{~nm}$ CdS layer is too thin to fully cover the CIGS NTRs, resulting in a much lower shunt resistance while the thickness of $150 \mathrm{~nm} \mathrm{CdS}$ layer is too thick to degrade the performance of efficiency because of larger series resistance. As a result, the best thickness of CdS layer in current study is $100 \mathrm{~nm}$, yielding the highest efficiency of $\sim 5.2 \%$.

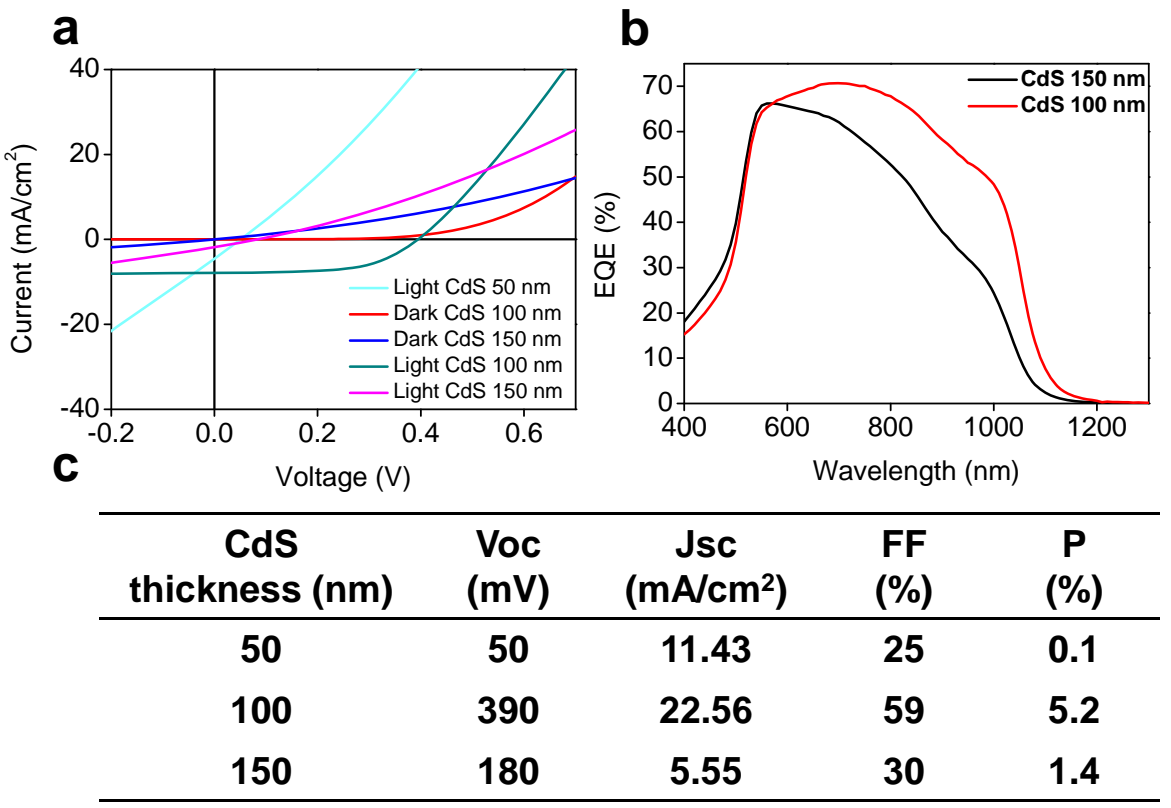

Figure S9: a, Dark and Light I-V measurements of CIGS NTRs solar cells with thickness of CdS buffer layers at 50,100, and $150 \mathrm{~nm}$, respectively. b, External quantum efficiency measurements of CIGS NTRs solar cells with CdS layers of 100 and $150 \mathrm{~nm}$, respectively, at excited wavelength ranged from 400 to $1300 \mathrm{~nm}$. c, The corresponding $\mathrm{V}_{\mathrm{oc}}, \mathrm{J}_{\mathrm{sc}}$, Efficiency, and filling factor of CIGS NTRs solar cells with different CdS layers from 50 to $150 \mathrm{~nm}$. The efficiency of each device is $0.1,5.2$ and $1.4 \%$, with FF of 23, 59 , and $30 \%$, respectively. 


\section{References}

1 Ogawa, Y., Jäger-Waldau, A., Hua, T. H., Hashimoto, Y. \& Ito, K. Influence of KCN treatment on $\mathrm{CuInS}_{2}$ thin films. Applied Surface Science 1996, 92, 232-236.

2 Klenk, R.; Menner, R.; Cahen, D.; Schock, H. W. In. Improvement of Cu(Ga,In)Se $\mathrm{Se}_{2}$ based solar cells by etching the absorber, Photovoltaic Specialists Conference, 1990, 1, 481-486. 